\title{
Rationale for the autologous serum skin test in acute versus chronic urticaria
}

\author{
Serkan Demirkan ${ }^{1}$, Ayşe Baççıoğlu² \\ 1Department of Dermatology and Venerology, Faculty of Medicine, Kirikkale University, Kirikkale, Turkey \\ 2Department of Pulmonary Diseases, Division of Immunology and Allergy, Faculty of Medicine, Kirikkale University, Kirikkale, Turkey \\ Adv Dermatol Allergol 2019; XXXVI (6): 703-706 \\ DOI: https://doi.org/10.5114/ada.2019.91421
}

\begin{abstract}
Introduction: Autologous serum skin test (ASST) is a rapid, in-vivo clinical test to detect functional autoantibodies in patients with chronic spontaneous urticaria (CSU), but the rationale for its use in acute urticaria (AU) is unknown. Aim: To evaluate the efficacy of ASST among patients with AU or CSU.

Material and methods: Treatment-naïve adult (age $\geq 18$ years) patients with a diagnosis of AU ( 6 weeks' duration) and CSU were enrolled prospectively in a cross-sectional study. Healthy age- and sex-matched subjects served as controls. Besides a detailed history and physical examination, ASST, total immunoglobulin E (IgE), freeT3 (fT3), freeT4 (fT4), anti-thyroglobulin, and anti-TPO levels were assessed in all subjects.

Results: Of 101 subjects, mean age was $34.35 \pm 12.68$ years and the study comprised $58.4 \%$ of females with no difference between AU $(n=27)$, CSU $(n=46)$, and control groups $(n=28)$. The ratio of positivity in ASST was similar between $\mathrm{AU}(25.9 \%)$ and CSU groups (21.7\%), but higher than in controls (10.7\%, $p=0.33$ for all). The ratio of patients with high total IgE levels $(>100 \mathrm{IU} / \mathrm{ml})$ in $\mathrm{AU}(85.2 \%)$ and CSU (65.2\%) groups was similar $(p=0.06)$, but significantly higher than in the control group (10.7\%) $(p<0.001$ and $p<0.001)$. The CSU group had significantly higher abnormal thyroid test results (45.7\%) than AU (14.8\%) and control groups (3.6\%) ( $p=0.01$ and $p<0.001)$, whereas patients with clinically diagnosed thyroiditis were only in the CSU group (6.5\%). In logistic regression analysis, there was no relation found among the possible risk factors for ASST, even if analysed separately as AU, CSU and control groups. Conclusions: Even though thyroid function test levels were found to be related with CSU, and total IgE was associated with urticaria, ASST was found to be of importance. This study confirms that ASST was insufficient to demonstrate autoimmunity and acute-chronic urticaria nature. Further tests indicating auto-antibodies in AU and CSU are needed.
\end{abstract}

Key words: autologous serum skin test, chronic urticaria, autoimmunity.

\section{Introduction}

Autologous serum skin test (ASST) is an intradermal application of the patient's serum sample to detect autoantibodies against high-affinity immunoglobulin E (IgE) receptor, or IgE with a high sensitivity and specificity of about $80 \%$ [1]. Positivity in ASST with an induration larger than $1.5 \mathrm{~mm}$ indicates autoimmunity in urticaria. This rapid in vivo test may help to detect circulating functional auto-antibodies, and thus may show the need of immunomodulatory therapy [2]. The incidence of ASST positivity in patients with chronic spontaneous urticaria (CSU) is reported to be in a larger range, i.e. between $4.1 \%$ and $82.1 \%$, which might be due to different criteria for positivity [1-3]. Although the general aspect is that ASST is not useful in diseases other than CSU, recent studies have shown ASST positivity in patients with asthma, rhinitis, multidrug allergy syndrome and healthy individuals, at a ratio of up to $55.55 \%$ [2, 4-7]. Acute urticaria (AU) which lasts shorter than 6 weeks, has identifiable aetiology only in $50 \%$ of patients. General laboratory studies are not indicated in $\mathrm{AU}$, however etiologic examination including ASST was shown to presume progression toward CSU [8].

\section{Aim}

The aim of this study was to determine the rationality of ASST in AU in comparison with CSU and healthy

Address for correspondence: Serkan Demirkan MD, Department of Dermatology and Venerology, Faculty of Medicine, Kirikkale University, Yenisehir District, Tahsin Duru Avenue 14, Yahsihan, Kirikkale, Turkey, phone: +90 5537717168, fax: +90 3182444697, e-mail: serkan.demirkan@yahoo.com.tr

Received: 16.01.2018, accepted: 11.07.2018. 
adults. We hypothesized that ASST is useful to identify aetiology not only in CSU, but also in AU.

\section{Material and methods}

This study was a prospective cross-sectional study carried out between 2016 and 2017. It was approved by the Local Ethical Committee of the Kirikkale University (2016/11/08). Patients were recruited from the Departments of Dermatology and Allergy in the Kirikkale University Hospital.

The diagnosis of AU and CSU was done according to the criteria of the European Academy of Allergy and Clinical Immunology (EAACI) [1]. Recruitment criteria for patients included: 1) untreated urticaria patients, 2) diagnosed by an expert with a specialty in dermatology or allergy, 3) if the patient had a history of recurrent wheals over 6 weeks he/she was grouped as CSU, or vice versa, 4) the patients had no definite causes in history such as allergic diseases, autoimmune diseases, infections, or drug allergy evidence. Exclusion criteria were: 1) identified urticaria reason such as physical, cholinergic, vasculitis or hereditary angioedema, 2) using antihistamine within a week, steroid or immunosuppressive drugs within a month, 3) having an exact cause of urticaria during the study follow-up, 4) pregnant or nursing women were excluded. In AU, AST was not performed until the symptoms completely subsided.

Clinical details of all subjects were recorded. Selected patients were subjected to ASST and laboratory investigations. The ASST was performed with $0.5 \mathrm{ml}$ of the patient's own serum injected intradermally into the flexor aspect of the forearm; $0.5 \mathrm{ml}$ of saline was injected 3 to
$5 \mathrm{~cm}$ away as a control. The test was considered positive if the diameter of the papule after $30 \mathrm{~min}$ was $1.5 \mathrm{~mm}$ or greater than that of the saline papule [9].

Anti-thyroglobulin, antithyroid peroxidase (anti-TPO), free (f) T3, fT4, and TSH tests were also performed as autoimmunity indicators in addition to total immunoglobulin (Ig) gE tests in Immunoassay Analyzer (Roche, Basel, Switzerland). Deficiency levels of total $\mathrm{lgE}(<100 \mathrm{IU} / \mathrm{ml})$, sTSH $(0.5-5 \mu \mathrm{U} / \mathrm{ml})$, fT3 (2.2-4 pg/ml), fT4 (0.76-1.46 ng/ $\mathrm{ml})$, anti-thyroglobulin $(<40 \mathrm{lU} / \mathrm{ml})$, anti-TPO $(<35 \mathrm{lU} / \mathrm{ml})$ were determined according to the manufacturer's standards. Abnormality in thyroid tests was defined as any thyroid test out of the normal range. A patient with clinical thyroiditis was defined as with abnormal TSH, fT3 and fT4 levels besides high levels of thyroid antibodies.

\section{Statistical analysis}

The data were recorded and processed using SPSS 17.0 (SPSS Inc., Chicago, IL, USA). The positive rates are expressed as percentages and were analysed using the $\chi^{2}$ test. Numeric variables are expressed as means and were analysed using the Student's T-test. A $p$-value $<0.05$ was considered significant.

\section{Results}

The study group was composed of adults with a mean age of $34.35 \pm 12.68$ years, and a female dominance $(58.4 \%)$ (Table 1 ). Patients were treatment naïve except $8.7 \%$ of the CSU group who used anti-allergic drugs long before admission.

Table 1. Characteristics of patient and control groups $(N=101)$

\begin{tabular}{|c|c|c|c|c|c|}
\hline Parameter & $\begin{array}{c}\text { All } \\
(n=101) \\
\%(n) \text { or } \\
\text { mean } \pm \text { SD }\end{array}$ & $\begin{array}{l}\text { Acute urticaria } \\
\qquad(n=27) \\
\%(n) \text { or } \\
\text { mean } \pm \text { SD }\end{array}$ & $\begin{array}{l}\text { Chronic idiopathic urticaria } \\
\qquad(n=46) \\
\%(n) \text { or } \\
\text { mean } \pm \text { SD }\end{array}$ & $\begin{array}{l}\text { Control group } \\
\quad(n=28) \\
\%(n) \text { or } \\
\text { mean } \pm \text { SD }\end{array}$ & $P$-value \\
\hline Gender, female & $58.4(59)$ & $74.1(20)$ & $50(23)$ & $57.1(16)$ & 0.13 \\
\hline Age [years] & $34.35 \pm 12.68$ & $32.96 \pm 12$ & $37.17 \pm 15.05$ & $31.07 \pm 7.27$ & 0.16 \\
\hline Previously under treatment & & - & $8.7(4)$ & - & - \\
\hline
\end{tabular}

Table 2. Laboratory examination results (mean \pm standard deviation)

\begin{tabular}{|c|c|c|c|c|}
\hline Parameter & $\begin{array}{l}\text { Acute urticaria } \\
\qquad(n=27)\end{array}$ & $\begin{array}{l}\text { Chronic idiopathic urticaria } \\
\qquad(n=46)\end{array}$ & $\begin{array}{l}\text { Control group } \\
\quad(n=28)\end{array}$ & $P$-value \\
\hline Total lgE [IU/ml] & $247.88 \pm 19.79$ & $250.87 \pm 37.46$ & $75.67 \pm 18.07$ & $<0.001$ \\
\hline $\mathrm{sTSH}[\mu \mathrm{U} / \mathrm{ml}]$ & $1.47 \pm 0.52$ & $3.34 \pm 1.45$ & $1.41 \pm 0.39$ & 0.27 \\
\hline fT3 [pg/ml] & $3.34 \pm 0.38$ & $3.38 \pm 0.42$ & $3.45 \pm 0.26$ & 0.55 \\
\hline fT4 [ng/ml] & $1.41 \pm 0.21$ & $1.14 \pm 0.20$ & $1.38 \pm 0.18$ & $<0.001$ \\
\hline Anti-thyroglobulin [IU/ml] & $14.97 \pm 3.59$ & $46.85 \pm 13.06$ & $10.76 \pm 2.82$ & 0.022 \\
\hline Anti-TPO [IU/ml] & $19.07 \pm 6.61$ & $53.86 \pm 13.70$ & $5.17 \pm 0.92$ & 0.006 \\
\hline
\end{tabular}

sTSH - sensitive TSH, fT3 - free T3, fT4 - free T4. 
Mean serum total IgE levels were significantly higher in patients with urticaria than the control group, but were similar between AU and CSU groups. Mean fT4, anti-thyroglobulin, and anti-TPO levels in the CSU group were higher than both $\mathrm{AU}$ and control groups (Table 2).

The ratio of ASST positivity was insignificantly higher in the $\mathrm{AU}(25.9 \%)$ and CSU group (21.7\%) than in the control group (10.7\%) (Figure 1). When serum test values were defined as abnormal according to normal limits, the results were also similar as mentioned above. In logistic regression analysis, no significant risk factor was determined for ASST positivity even if analysed separately in groups.

\section{Discussion}

The gold standard for the diagnosis of autoimmunity in CSU is to find positivity in these three tests; ASST positivity (auto-reactivity), bioassay positivity (basophile histamine release assay or basophile activation indicator expression), and immunoassay positivity (Western blot or ELISA immunoreactivity) [2]. However, it is not a useful method to be applied to all CSU patients in practical life, since they need specialized laboratories.

\section{Frequency of ASST positivity}

ASST positivity in patients with CSU was reported between $4.1 \%$ and $82.1 \%$, with different methods for positivity criteria [1-3]. In this study, the ratio of ASST positivity was almost one fourth of the urticaria patients and one tenth of healthy ones. Even though these results were lower than in some studies, similarity between urticaria and healthy subjects was a frequent result as in other articles. For example, Taşkapan et al. found that its positivity was 53\% in CSU patients, $20 \%$ in patients with allergic rhinitis/asthma, and $56 \%$ in healthy controls [2]. Furthermore, this was one of the first studies comparing ASST positivity among AU and CSU patients. In PubMed search with key words of AU and ASST, there was only one research paper published by Magen et al. [8]. They reported ASST positivity as 63.4\% in the CSU, and $23.3 \%$ in the AU group. However, this significant difference disappeared after 7 weeks of survey. The contrary results between the two studies may be attributed to different inclusion criteria. As we know this is the first study with patient enrolment from two different departments that deal with urticaria, and this side of the study makes sense if the whole population was included. We suggested that urticaria patients who were admitted to the Allergy Department had a suspicion of allergy, especially to drugs, whereas patients in the Dermatology Department were reported to have autoimmunity in a published paper.

\section{Reliability of ASST}

In this study, ASST was found to be insufficient to differentiate autoimmunity in patients from healthy subjects. Actually, it has been known that ASST is not

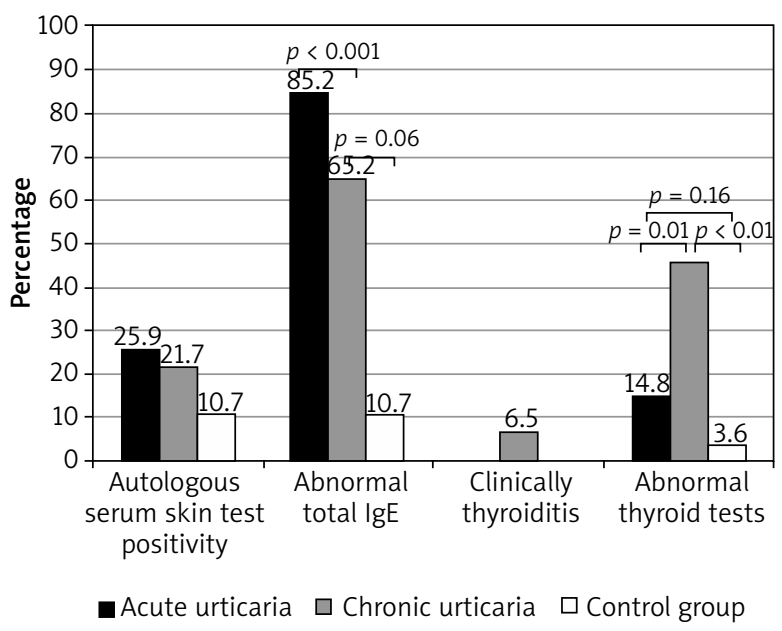

Figure 1. Comparison of autologous serum skin test positivity, abnormality in serum total IgE levels and thyroid tests between acute and chronic urticaria groups, and the control group

specific for CSU. For example, Güven et al. found ASST positivity in patients with alopecia and vitiligo [10]. Its sensitivity and specificity was reported as approximately 70-80\% [11]. It has been reported that this test was dependent on histamine and mast cell response, and can be passively transferred to healthy individuals. However, it is controversial whether circulating immune complexes are responsible for this response since only $50 \%$ of the patients with CSU had autoantibodies filled up [12, 13]. Likewise, heating the serum to a certain temperature at which IgE was inactivated, did not change the positivity of the ASST $[12,14-16]$. In this case, it would not be appropriate to consider urticaria patients with positive ASST as autoimmune ones [17-19]. Furthermore, in some studies, there was no relationship between ASST and treatment response or disease course [19]. These were the points that make the reliability of ASST doubtful.

\section{Reproducibility of ASST}

There are controversial reports advocating that the ASST is a reproducible test [16, 20-23]. Magen et al. observed that there was a conversion in ASST results of patients with CSU in follow-ups as positivity to negativity or vice versa. On the other hand, Eryılmaz et al. argued that ASST should be repeated several times to use it as a screening test for autoimmune urticaria [24]. Even though there was no follow-up in this study, the similarity of ASST results between AU, CSU, and healthy groups would suggest that there would be no change if patients with $\mathrm{AU}$ were followed up. We concluded that ASST was not rational in patients with CSU. ASST shows neither diagnostic value for autoimmunity nor reflects urticaria activity. It may be considered that ASST does not contribute to disease management. 


\section{Thyroid function test and total IgE results}

Studies suggesting the role of thyroid autoimmunity and its relation with ASST in CSU are controversial. In some, ASST positivity was found to be higher in CSU patients that had thyroid autoantibodies than patients without [13, 25, 26]. Furthermore, an exact relationship in CSU patients between having higher autoimmune disease with determined autoantibody and ASST positivity was written in guidelines [1], even though there were opposite results advocating usefulness of ASST in showing autoimmunity [27, 28]. Finally, it was emphasized to look at thyroid autoantibodies although there was no significant difference of ASST between the CSU group and the control group [29]. Likewise, this study showed that clinically thyroiditis and/or abnormality in thyroid tests was significantly higher in the CSU group than the AU and healthy groups. Thus, we recommend performing routine thyroid autoantibodies and thyroid functional tests in CSU.

In this study, total IgE levels were significantly higher in urticaria patients than in the healthy group, which made us to think that patients of urticaria were rich of IgE levels as a target for mast or basophile cell mediator release. However, lgE levels are found to be unimportant to differentiate between AU and CSU patients.

\section{Conclusions}

Even though thyroid function tests were found to be related with CSU, and total IgE was associated with urticaria, ASST was found to be of importance. This study confirms that ASST is insufficient to demonstrate autoimmunity and acute-chronic urticaria nature. Further research about auto-antibodies in AU and CSU is needed.

\section{Conflict of interest}

The authors declare no conflict of interest.

\section{References}

1. Konstantinou GN, Asero R, Maurer M, et al. EAACI/GA(2)LEN task force consensus report: the autologous serum skin test in urticaria. Allergy 2009; 64: 1256-68.

2. Taskapan O, Kutlu A, Karabudak O. Evaluation of autologous serum skin test results in patients with chronic idiopathic urticaria, allergic/non-allergic asthma or rhinitis and healthy people. Clin Exp Dermatol 2008; 33: 754-8.

3. Mete N, Gülbahar O, Sin A, et al. Kronik idiopatik ürtikerde otolog serum testi. Ege Tıp Dergisi 2003; 42: 25-9.

4. Taskapan O, Kutlu A. Autologous serum skin test: is the paradigm falling? J Eur Acad Dermatol Venereol 2009; 23: 956-7.

5. Guttman-Yassky E, Bergman R, Maor C, et al. The autologous serum skin test in a cohort of chronic idiopathic urticarial patients compared to respiratory allergy patients and healthy individuals. J Eur Acad Dermatol Venereol 2007; 21: 35-9.

6. Asero R, Tedeschi A, Lorini M. Autoreactivity is highly prevalent in patients with multiple intolerances to NSAIDs. Ann Allergy Asthma Immunol 2002; 88: 468-72.

7. Asero R, Tedeschi A, Lorini M, et al. Sera from patients with multiple drug allergy syndrome contain circulating histaminereleasing factors. Int Arch Allergy Immunol 2003; 131: 195-200.
8. Magen E, Zueva E, Mishal J, Schlesinger M. The clinical and laboratory characteristics of acute spontaneous urticaria and its progression to chronic spontaneous urticaria. Allergy Asthma Proc 2016; 37: 394-9.

9. Taşkın B, Eriş Eken Z, Alper S. The diagnosis and treatment approches in chronic spontaneous urticaria. Turkiye Klinikleri J Dermatol-Special Topics 2015; 8: 20-5.

10. Güven M, Gürler A, Erdoğan FM, Gündüz Ö. Positivity of autologous serum skin test in patients with alopecia areata and vitiligo and in healthy individuals. Turkderm 2015; 49: 184-90.

11. Stitt J, Dreskin S. Urticaria and autoimmunity: where are we now? Curr Allergy Asthma Rep 2013; 13: 555-62.

12. Sabroe RA, Greaves MW. Chronic idiopathic urticarial with functional autoantibodies: 12 years on. Br J Dermatol 2006; 154: 813-9.

13. Kaya Ti, Akyol A. Pathogenesis of urticaria: recent advances in the subject of chronic idiopathic urticarial pathogenesis. Turkiye Klinikleri Dermatol 1999; 9: 41-50.

14. Bakos N, Hillander M. Comparison of chronic autoimmune urticarial with chronic idiopathic urticaria. Int I Dermatol 2003; 42: 613-5.

15. Grattan CEH, Sabroe RA, Greaves MW. Chronic urticaria. J Am Acad Dermatol 2002; 46: 645-57.

16. Sabroe RA, Grattan CEH, Francis DM, et al. The autologous serum skin test: a screening test for autoantibodies in chronic idiopathic urticaria. Br J Dermatol 1999; 140: 446-52.

17. Nettis E, Dambra P, D’Oronzio L, et al. Reactivity to autologous serum skin test and clinical features in chronic idiopathic urticaria. Clin Exp Dermatol 2002; 27: 29-31.

18. Lapolla W, Desai N, English JC $3^{\text {rd }}$. Clinical utility of testing for autoimmunity in chronic idiopathic urticaria. J Am Acad Dermatol 2012; 66: 83-8.

19. Caproni M, Giomi B, Volpi W, et al. Chronic idiopathic urticaria: infiltrating cells and related cytokines in autologous seruminduced wheals. Clin Immunol 2005; 114: 284-2.

20. Aytekin S, Türkmen H. Investigation of autologous serum skin test and etiological factors in 31 patients with chronic urticaria. Turkiye Klinikleri Dermatol 2001; 11: 141-5.

21. Fagiolo U, Kricek F, Ruf C, et al. Effects of complement inactivation and IgG depletion on skin reactivity to autologous serum in chronic idiopathic urticaria. J Allergy Clin Immunol 2000; 106: 567-72.

22. Fusari A, Colangelo C, Bonifazi F, Antonicelli L. The autologous serum skin test in the follow-up of patients with chronic urticaria. Allergy 2005; 60: 256-8.

23. Grattan CEH, Wallington TB, Warin RP, et al. A serological mediator in chronic idiopathic urticaria - a clinical, immunological and histological evaluation. Br J Dermatol 1986; 114: 583-90.

24. Eryılmaz A, Aksungur VL, Baba M. The course of autologous serum skin test in chronic urticaria. Turkderm 2004; 38: 257-63.

25. Mete N, Gulbahar O, Aydin A, et al. Low B12 levels in chronic idiopathic urticaria. J Investig Allergol Clin Immunol 2004; 14: 292-9.

26. Huber J, Hawkes JE, Powell DL. Cobalt-Induced contact urticaria presenting as chronic urticaria due to intramuscular vitamin B12 injections. Dermatitis 2016; 27: 313-4.

27. Leznoff A, Sussman GL. Syndrome of idiopathic chronic urticarial and angioedema with thyroid autoimmunity. J Allergy Clin Immunol 1989; 84: 66-71.

28. Pokhrel K, Subedi S, Acharya S. Autologous serum skin test in chronic idiopathic urticaria. JNGMC 2014; 12: 6-10.

29. Alpay A, Solak Tekin N, Özel Tekin I, et al. Autologous serum skin test versus autologous plasma skin test in patients with chronic spontaneous urticaria. Dermatol Res Prat 2013; 2013: 267278. 